\title{
EXAMINING THE EFFECTS OF SUCROSE ON INFANT PHYSIOLOGY
}

\author{
B. Tam, R. Slater, L. Fabrizi, J. Meek \\ UCL, London, $U K$
}

\begin{abstract}
An experimental study examining the effects of sucrose as an analgesic for newborn infants, showed significant differences in the Premature Infant Pain Profile (PIPP) scores between infants administered sucrose and sterile water but not on electroencephlography (EEG) measures of nociceptive central nervous system activity. The aim of this study was to investigate which components of the PIPP (physiological response, facial response and behavioural state) contributed to the significant between-group differences.
\end{abstract}

A secondary analysis was performed on the data published in the sucrose RCT (Slater et al., Lancet, 2010). $t$ tests were performed to test for mean differences between the groups on the individual components of the PIPP. Partial correlations were conducted to examine the correlations between the PIPP subscales and EEG measures after controlling for experimental condition (sucrose/sterile water).

Significant differences were found on the PIPP physiological subscale between the sucrose $(\mathrm{M}=1.3$; $S D=1.26)$ and sterile water groups $(\mathrm{M}=2.33 ; S D=1.31 ; t(42)=-2.653, p<0.05)$. There were no significant differences between the facial expression scores or the behavioural state scores between the groups. After controlling for experimental condition (sucrose/sterile water), no significant partial correlation was found between any PIPP subscale and EEG measure.

When the PIPP score is separated into its component parts the significant difference observed between the two groups can be attributed to group differences in the physiological score. This implies that sucrose is stabilising infant physiology rather than modulating nociceptive-specific CNS activity. 